\title{
MUDANÇAS CLIMÁTICAS: DESAFIOS E OPORTUNIDADES PARA A CONSERVAÇÃO DA BIODIVERSIDADE BRASILEIRA
}

\author{
Mariana M. Vale ${ }^{l^{*}}$, Maria Alice S. Alves ${ }^{1}$ \& Maria Lucia Lorini ${ }^{2,3}$ \\ ${ }^{1}$ Departamento de Ecologia, Instituto de Biologia Roberto Alcantara Gomes, Universidade do Estado do Rio de Janeiro (UERJ). Rua São Fran- \\ cisco Xavier 524. Rio de Janeiro, Brasil. CEP: 20550-011. \\ ${ }^{2}$ Laboratório de Gestão da Biodiversidade, Dep. Botânica, Inst. de Biologia, Universidade Federal do Rio de Janeiro (UFRJ). Ilha do Fundão. Caixa \\ Postal: 68029. Rio de Janeiro, Brasil. CEP: 21941-971. \\ ${ }^{3}$ Programa de Pós-Graduação em Ecologia, Instituto de Biologia, UFRJ. Ilha do Fundão. Caixa Postal: 68029. Rio de Janeiro, Brasil. CEP: $21941-971$. \\ E-mails: mvale.eco@gmail.com,masaal@globo.com, marialucia.lorini@gmail.com.
}

\section{RESUMO}

A realidade das mudanças climáticas pelas quais o planeta está passando é inequívoca, assim como a influência humana nesse processo. O Painel Intergovernamental sobre Mudanças Climáticas das Nacões Unidas prevê mudanças climáticas substanciais para a região Neotropical. De fato, os impactos dessas mudanças climáticas sobre a biodiversidade da região já estão sendo sentidos: colapso de ecossistemas de corais no Caribe, retração de glaciares nos Andes e eventos extremos de seca na Amazônia. Embora haja um corpo substancial de conhecimento em torno dos possíveis impactos das mudanças climáticas sobre as formações florestais brasileiras, sobretudo as amazônicas, estudos dos impactos sobre elementos da biodiversidade do país são praticamente inexistentes. Nos últimos 20 anos foram publicados apenas dois artigos científicos em periódicos indexados na base do Thompson Institute for Scientific Information (ISI). Essa lacuna de conhecimento impossibilita a elaboração de quaisquer estratégias de adaptação às mudanças climáticas visando à conservação da biodiversidade brasileira. A comunidade científica e as agências de fomento à pesquisa, portanto, devem começar a priorizar estudos dessa natureza. O Brasil, no entanto, não deve se concentrar apenas em estratégias de 'adaptação', pois existe grande potencial no país para ações de 'mitigação' das mudanças climáticas em andamento. Apesar da sua matriz energética limpa, o país figura entre os maiores emissores mundiais de gases de efeito estufa, devido às enormes emissões associadas ao desmatamento. Uma questão de primeira ordem é a histórica oposição do país à incorporação de 'desmatamento evitado' como um dos Mecanismos de Desenvolvimento Limpo do Protocolo de Kyoto. É importante que a comunidade científica brasileira ligada à conservação participe ativamente desse debate, cujas conseqüências para a biodiversidade são bastante graves.

Palavras-chaves: Protocolo de Kyoto, adaptação, mitigação, Neotrópico, Brasil.

\begin{abstract}
CLIMATE CHANGE: CHALLENGES AND OPPORTUNITIES FOR BIODIVERSITY CONSERVATION IN BRAZIL. The reality of the climatic changes that the planet is going through is unequivocal, and so is the human influence in this process. The Intergovernmental Panel on Climate Change of the United Nations predicts substantial climatic changes for the Neotropical region. In fact, the impact of these climatic changes over the region's biodiversity is already being felt: collapse of coral ecosystems in the Caribbean, glaciers retraction in the Andes, and extreme drought events in the Amazon. Although there is a substantial body of knowledge about the possible impacts of climate change over the Brazilian forest formations, especially in the Amazon, studies about the impacts on elements of the countries' biodiversity are virtually inexistent. In the last 20 years only two scientific papers were published in indexed journals within the Thompson Institute for Scientific Information (ISI) database. This knowledge gap prevents the elaboration of any adaptation strategy
\end{abstract}


aiming at the conservation of biodiversity in Brazil. The scientific community and funding agencies, therefore, must start prioritizing that brand of studies. Brazil, however, should not concentrate only on adaptation strategies, because there already is a great potential for mitigation of the ongoing climatic changes. Despite of its relatively clean energetic network, the country is among the World's greatest emitters of greenhouse gases because of the enormous emissions associated with deforestation. A first order issue is the countries' historical opposition to the incorporation of 'avoided deforestation' as one of the Clean Development Mechanisms within the Kyoto Protocol. An active participation of the scientific community linked to conservation this debate is paramount, as it has serious consequences for biodiversity conservation in Brazil.

Key-words: Kyoto Protocol, adaptation, mitigation, Neotropics, Brazil.

\section{RESUMEN}

CAMBIO CLIMÁTICO: DESAFÍOS Y OPORTUNIDADES PARA LA CONSERVACIÓN DE LA BIODIVERSIDAD BRASILEÑA. La realidad del cambio climático por el que está pasando el planeta es inequívoca, así como la influencia humana en este proceso. El Panel Intergubernamental sobre Cambio Climático de las Naciones Unidas prevé cambios climáticos sustanciales para la región Neotropical. De hecho, los impactos de estos cambios climáticos sobre la biodiversidad de la región ya se están sintiendo: colapso de los ecosistemas de coral en el Caribe, retroceso de glaciares en los Andes y eventos extremos de sequía en la Amazonia. Aunque hay una cantidad importante de conocimiento sobre los posibles impactos de los cambios climáticos sobre las formaciones boscosas brasileñas, principalmente las amazónicas, estudios de los impactos sobre elementos de la biodiversidad del país son prácticamente inexistentes. En los últimos 20 años fueron publicados sólo dos artículos científicos en revistas indexadas en la base de datos Thompson Institute for Scientific Information (ISI). Este vacío de conocimiento imposibilita la elaboración de cualquier estrategia de adaptación al cambio climático que apunte a la conservación de la biodiversidad brasileña. Por esto, la comunidad científica y las agencias de fomento a la investigación deben comenzar a dar prioridad a estudios de esta naturaleza. El Brasil, sin embargo, no se debe concentrar únicamente en estrategias de 'adaptación', pues existe gran potencial en el país para acciones de 'mitigación' de los cambios climáticos actuales. A pesar de su matriz energética limpia, el país figura entre los mayores emisores mundiales de gases de efecto invernadero, debido a las enormes emisiones asociadas a la deforestación. Un asunto de primer orden es la histórica oposición del país a la incorporación de la 'deforestación evitada' como uno de los Mecanismos de Desarrollo Limpio del Protocolo de Kyoto. Es importante que la comunidad científica brasileña vinculada a la conservación participe activamente de este debate, cuyas consecuencias para la biodiversidad son bastante graves.

Palabras-claves: Protocolo de Kyoto, adaptación, mitigación, Neotropico, Brasil.

\section{A REALIDADE DAS MUDANÇAS CLIMÁTICAS}

Recentemente uma sucessão de eventos tornou as mudanças climáticas globais uma questão de primeira ordem: o lançamento do filme do senador americano $\mathrm{Al}$ Gore (http://www.climatecrisis.net), a divulgação do relatório do Ministério do Meio Ambiente sobre Mudanças Climáticas Globais e seus Efeitos sobre a Biodiversidade (Marengo 2007), do 'Relatório Stern' (Stern 2007) e, sobretudo, do $4^{\circ}$ Relatório do Painel Intergovernamental de Mudanças Climáticas da Organização das Nações Unidas (ONU) (IPCC 2007a). Por seu trabalho, o Painel Intergovernamental de Mudanças Climáticas (IPCC, na sigla em inglês) e Al Gore foram agraciados com o prêmio Nobel da Paz de 2007. Essa foi a primeira representação da área ambiental no prestigioso prêmio. No Brasil, esses eventos refletiram-se na formação oficial de uma Comissão Mista Especial sobre Mudanças Climáticas, criada em 2007, para acompanhar, monitorar e fiscalizar as ações referentes às mudanças climáticas no Brasil. Um dos produtos desta comissão foi o Relatório Final (Brasil/CN/CME 2008), que reúne os trabalhos de mais de um ano do colegiado, sugerindo algumas medidas concretas para a mitigação e adaptação aos efeitos das mudanças climáticas. Esse trabalho evidencia um engajamento do Congresso 
Nacional brasileiro no debate, o que é louvável uma vez que a realidade das mudanças climáticas pelas quais o planeta está passando é inequívoca, assim como a influência humana nesse processo.

O relatório do IPCC revela que as mudanças climáticas estão bem mais aceleradas do que se havia previsto e que as suas conseqüências já estão sendo sentidas. Isso não é uma novidade para a comunidade científica que já vinha publicando resultados nesse sentido (Walther et al. 2005). De fato, foi justamente um acúmulo de evidências que levou ao consenso atual sobre o tema. A produção de artigos científicos sobre mudanças climáticas, que era incipiente na década de 80 , vem apresentando um crescimento expressivo desde 1991 (Figura 1). De 1987 a 2007 a porcentagem anual da publicação sobre "mudanças climáticas", em relação ao total de artigos produzidos sobre a temática "clima", registrou um aumento de mais de 40 pontos percentuais. Em 2007, o ano da publicação do relatório do IPPC, a produção cumulativa associada ao tema das mudanças climáticas totalizou quase 35.000 artigos, considerando apenas periódicos científicos indexados na base ISI.
Para a vasta gama de cenários de emissão e modelos de circulação global, o IPCC prevê até o final do século XXI, um aquecimento médio global de 1,1 a $6,4^{\circ} \mathrm{C}$ e um aumento do nível do mar de 0,16 a $0,56 \mathrm{~m}$, com continentes aquecendo mais que oceanos e altas latitudes aquecendo mais que os trópicos (IPCC 2007a). A revisão do Ministério do Meio Ambiente prevê para o Brasil, até 2100, um aumento de cerca de $4^{\circ} \mathrm{C}$ na temperatura média, com projeções regionais para a Amazônia envolvendo elevação de até $8^{\circ} \mathrm{C}$ que, conjugada à redução de chuvas, levaria à substituição de florestas por savanas. No sul do Brasil projeta-se um aumento de eventos extremos como inundações e ondas de calor (Marengo 2007), nos moldes das catastróficas chuvas de novembro de 2008 em Santa Catarina. O estudo prevê também um impacto sobre até 42 milhões de habitantes do litoral brasileiro, embora esta estimativa tenha sido amplamente criticada (Leite 2007). É importante notar que as estimativas do IPCC são conservadoras, pois não incluem mecanismos de retroalimentação (como a savanização da Amazônia, por exemplo) na modelagem das mudanças climáticas (Meehl et al. 2007). A inclusão de mecanismos de

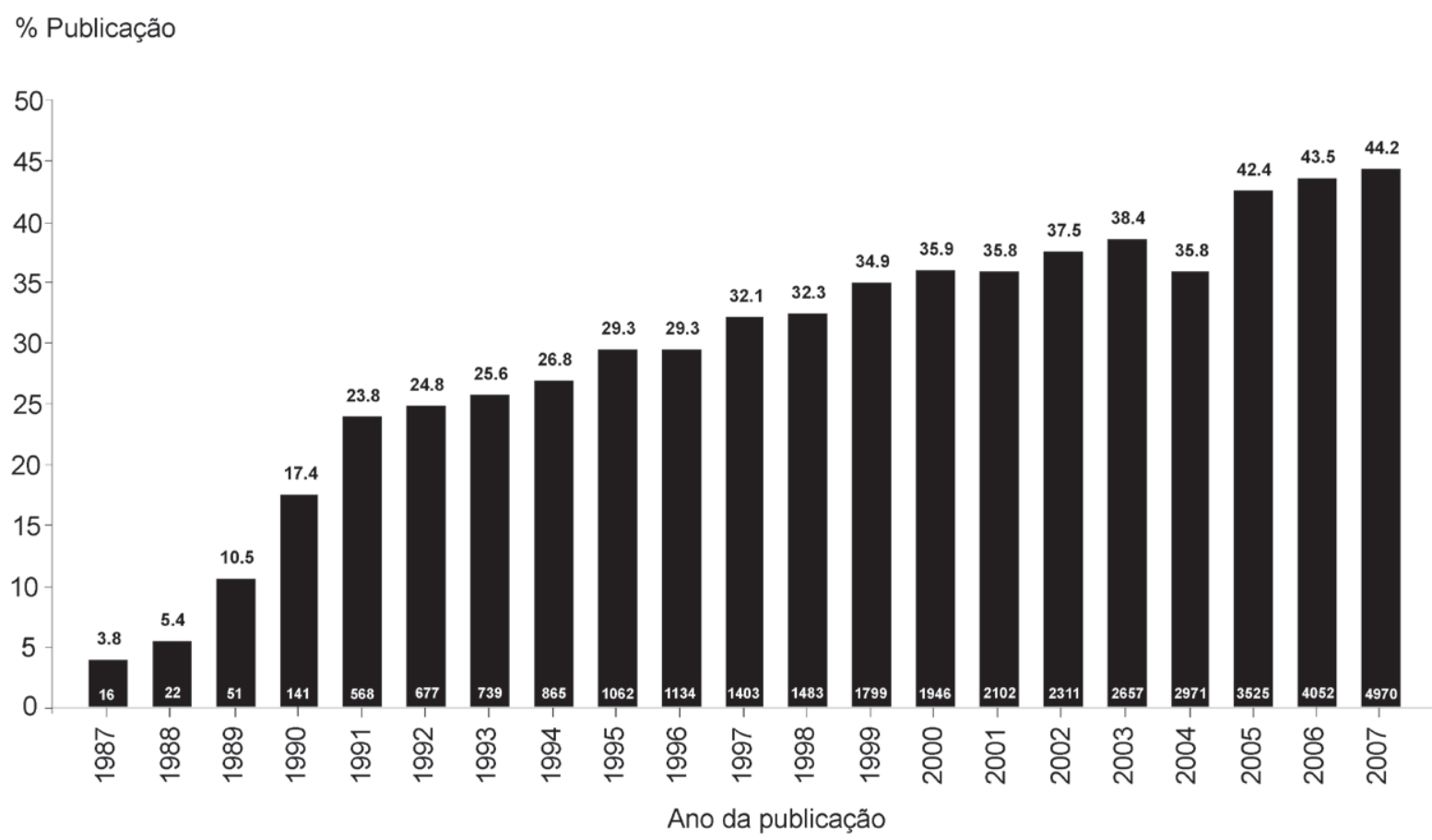

Figura 1. Tendência na publicação de artigos científicos associados ao tema 'mudança climática' ao longo dos últimos 20 anos $(n=34494)$, em relação aos referentes ao tema 'clima' $(n=95670)$. O número de artigos está representado em branco dentro das colunas. Os dados foram compilados a partir da base de dados Web of Science de publicações científicas do Thompson ISI (Thompson Institute for Scientific Information), através de uma busca por artigos publicados entre 1987 e 2007, utilizando os termos climat* change* e climat* como palavras-chave.

Figure 1. General trend of scientific articles published on the topic 'climate change'over the last 20 years $(n=34494)$ in comparison to articles published on the topic 'climate' $(n=95670)$. The number of articles is given in white in the columns. Data were compiled from the Web of Science database in Thompson ISI (Thompson Institute for Scientific Information), based on a search for articles published between 1987-2007 with 'climat* chang*' and 'climat*' as keywords. 
retroalimentação em 11 modelos testados aumentou a temperatura média global em $30 \%$ para o ano de 2100, em comparação com estimativas de modelos que não incluem esses mecanismos (Meehl et al. 2007, Fearnside 2008).

\section{MUDANÇAS CLIMÁTICAS E BIODIVERSIDADE}

Existe evidência convincente indicando que as mudanças climáticas ocorridas no século XX causaram vários impactos sobre a biodiversidade (Hughes 2000, McCarty 2001, Walther et al. 2002, Walther et al. 2005). Os efeitos documentados estão associados a alterações na distribuição de espécies (Thomas \& Lennon 1999, Parmesan \& Yohe 2003, Austin \& Rehfisch 2005, Hickling et al. 2005, Root et al. 2005, Walther et al. 2005, Wilson et al. 2005, Lavergne et al. 2006, Moritz et al. 2008) e na fenologia de espécies (Crick et al. 1997, Menzel \& Fabian 1999, Visser \& Holleman 2001, White et al. 2003, Zavaleta et al. 2003, Tøttrup et al. 2006), assim como no aumento do seu risco de extinção (Wilson et al. 2005, Franco et al. 2006, Parmesan 2006, Pauli et al. 2006, Pounds et al. 2006, Foden et al. 2007).

Em 2100, de acordo com o IPCC, os níveis de $\mathrm{CO}_{2}$ atmosférico estarão substancialmente mais elevados do que os registrados nos últimos 650.000 anos e as temperaturas estarão entre as mais elevadas dos últimos 740.000 anos (Fischlin et al. 2007). Portanto, os impactos das rápidas e substanciais mudanças climáticas induzidas pelo homem provavelmente terão conseqüências muito mais graves para a biodiversidade do planeta no século XXI. Várias projeções de impactos das mudanças climáticas sobre a biodiversidade em cenários futuros desenham um panorama preocupante (Kerr \& Packer 1998, Sala et al. 2000, Erasmus et al. 2002, Peterson et al. 2002, Pearson \& Dawson 2003, Meynecke 2004, Skov \& Svenning 2004, Thomas et al. 2004, Thuiller et al. 2005, MEA 2005, Levinsky et al. 2007). As estimativas do IPCC indicam que 20 a $30 \%$ das espécies estarão em alto risco de extinção no caso de um aumento de 2 a $3^{\circ} \mathrm{C}$ na temperatura.

É consenso que as mudanças climáticas podem desempenhar um papel fundamental em processos de erosão da biodiversidade no futuro. Isoladamente, as mudanças climáticas podem representar a ameaça mais ubíqua para a biodiversidade, uma vez que produzirão impactos mesmo em áreas que apresentam baixa alteração antrópica (Malcolm et al. 2006). Contudo, a maior ameaça para o futuro da biodiversidade terrestre parece residir na sinergia entre as mudanças climáticas aceleradas e outro vetor de mudança global, a modificação de paisagens, derivada, sobretudo, do uso do solo para agropecuária e urbanização (Sala et al. 2000, Root et al. 2003, Opdam \& Wascher 2004, MEA 2005, Lovejoy \& Hannah 2005, Thuiller et al. 2006, Jetz et al. 2007). O efeito combinado de rápidas alterações climáticas e substantiva diminuição e/ou fragmentação de hábitat exercerá impacto futuro sobre a distribuição e a persistência de espécies e populações, que responderão individual e idiosincraticamente, como constatado no passado (Graham et al. 1996, Williams et al. 2004, Moritz et al. 2008). Por sua vez, estas respostas complexas poderão acarretar alterações na composição de comunidades, na estrutura de ecossistemas, na distribuição destes sistemas, inclusive de biomas, resultando em mudanças que irão reverberar em múltiplas escalas espaço-temporais e níveis de organização biológica (Williams et al. 2004, Thuiller et al. 2006).

Em função dos graves impactos que as mudanças globais podem representar para a diversidade biológica e para os serviços dos ecossistemas (MEA 2005, Schröter et al. 2005), que são essenciais para a manutenção da humanidade, o conhecimento sobre a associação entre biodiversidade e mudanças climáticas torna-se uma forte e urgente demanda da sociedade. Ainda que a produção acadêmica associada à temática de biodiversidade represente menos de $2,5 \%$ do total de artigos publicados nos últimos 20 anos sobre mudanças climáticas, esta área emergente vem apresentando tendências crescentes desde 2004 (Figura 2). Enquanto no início da década de 90 a porcentagem anual da publicação nesta área oscilava entre $0,5 \mathrm{e}$ $1,5 \%$ do total de artigos produzidos sobre mudanças climáticas, em 2007 este percentual já ultrapassava 4\%. No período compreendido entre 1990 e 2007, a produção científica associando mudanças climáticas e biodiversidade já havia acumulado 847 artigos. Esta produção foi veiculada em 271 periódicos, com destaque para os títulos Global Change Biology, Biological Conservation, Journal of Biogeography, Biodiversity and Conservation, Conservation Biology, Global Ecology and Biogeography, que em conjunto compreenderam cerca de $20 \%$ do total de publicações. 


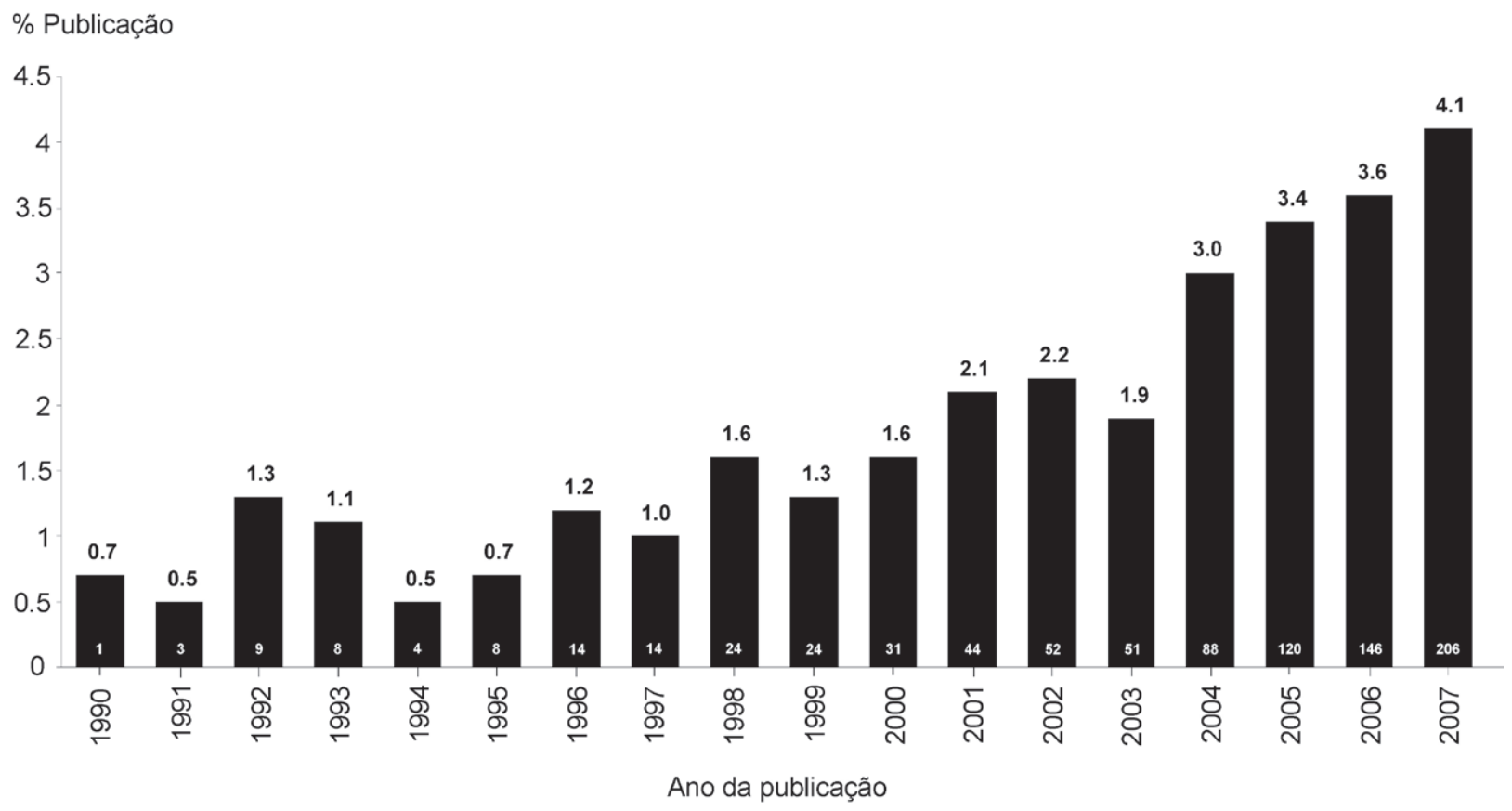

Figura 2. Tendência na publicação de artigos científicos relacionados à temática de 'mudança climática associada à biodiversidade' ao longo dos últimos 20 anos $(n=847)$, em relação aos referentes ao tema 'mudança climática' $(n=34494)$. O número de artigos está representado em branco dentro das colunas. Os dados foram compilados a partir da base de dados Web of Science de publicações científicas do Thompson ISI (Thompson Institute for Scientific Information), através de uma busca por artigos publicados entre 1987 e 2007 , utilizando os termos climat* change* mais biodiversity e climat* change* como palavras-chave.

Figure 2. General trend of scientific articles published on the topic 'climate change and biodiversity' over the last 20 years ( $n=847)$ in comparison to articles published on the topic 'climate change' $(n=34494)$. The number of articles is presented in white in the columns. Data were compiled from the Web of Science database in Thompson ISI (Thompson Institute for Scientific Information), based on a search for articles published between 19872007 with 'climat* chang*' plus 'biodiversity', and 'climat* chang*' as keywords.

De um modo geral, as espécies podem desenvolver quatro respostas às mudanças climáticas: dispersão, aclimatação, adaptação ou extirpação (Holt 1990, Peterson et al. 2001). Se a espécie apresentar grande habilidade de dispersão, potencialmente ela poderá abandonar áreas que se tornem inóspitas e colonizar áreas que se tornem favoráveis. Da mesma maneira, se a espécie conseguir se adaptar evolutivamente aos novos ambientes ou for plástica o suficiente para manter-se neles através de aclimatação, é provável que ela possa persistir na mesma área original. Por outro lado, se a espécie não apresentar habilidade para dispersão ou rápida adaptação, a probabilidade de extirpação (extinção local) será alta. Neste caso, restrições à adaptação e dispersão ao longo do novo espaço ambiental podem levar a espécie à extinção, dependendo de como estas características estejam ligadas à aptidão (Martínez-Meyer et al. 2004). Além disto, o tipo de resposta desenvolvido pela espécie pode em parte ser filogeneticamente estruturado, evoluindo sob diferentes modelos de evolução (Diniz-Filho \& Bini 2008). Em qualquer situação, cabe destacar que até mesmo as distâncias de dispersão podem modificar-se em resposta às mudanças climáticas (Møller et al. 2006).

Sem dúvida uma das principais questões encaradas pela pesquisa sobre mudanças climáticas e biodiversidade é saber em que extensão as espécies serão capazes de dispersar para os seus novos hábitats favoráveis (McKenney et al. 2007). As futuras distribuições serão determinadas não apenas pelo clima, mas também por uma hierarquia de fatores, incluindo outros fatores abióticos (por exemplo: topografia, condições do solo, etc.), bem como capacidade de dispersão dos organismos, interações bióticas (por exemplo competição, predação, etc.) e adaptação genética (McKenney et al. 2007). Contudo, diferente do que aconteceu em episódios de mudanças climáticas no passado, estes fatores estarão atuando em uma escala temporal extremamente curta e acelerada e, sobretudo, em paisagens altamente modificadas pelo homem.

Em termos de resposta de adaptação, o caso do chapim-real (Parus major) é ilustrativo. Devido ao 
aumento da temperatura, esta ave está vivendo um descompasso entre o período reprodutivo e o período de disponibilidade de larvas para alimentar os filhotes (Visser et al. 1998). A resposta evolutiva esperada seria o adiantamento do período reprodutivo, mas a espécie parece não estar reagindo. Respostas ecológicas de dispersão, como a redistribuição geográfica para acomodar mudanças climáticas, também não são triviais. Simulações executadas com base em modelagem de nicho destacam que a realização geográfica do nicho climático de muitas espécies será deslocada em direção a maiores latitudes e maiores elevações (Bakkenes et al. 2002, Thomas et al. 2004, Thuiller et al. 2005). Estas mudanças de distribuição podem ser tão grandes que muitos autores questionam se as espécies serão capazes de migrar suficientemente rápido para acompanhar o deslocamento espacial de seus limites climáticos (Higgins et al. 2000, Davis \& Shaw 2001, Malcolm et al. 2002, Midgley et al. 2007). Uma projeção da redistribuição de espécies em um cenário pós-mudanças climáticas globais, cobrindo um quinto da área terrestre do planeta, prevê que 15 a $37 \%$ das espécies deverão estar ameaçadas de extinção até 2050 (Thomas et al. 2004). Obviamente, algumas espécies expandirão suas populações com as mudanças climáticas em andamento. É o caso de duas espécies de borboletas na Inglaterra que estão ampliando sua área de distribuição devido ao aumento da temperatura (Thomas et al. 2001), causa para celebração em um país de pequena biodiversidade. A questão que se impõe é: podemos esperar que o mesmo aconteça nos trópicos? As áreas temperadas são caracterizadas por espécies de ampla distribuição geográfica. Já os trópicos, onde está a maior parte da diversidade do planeta, são caracterizados por espécies de distribuição restrita. Portanto, no jogo de azar do aquecimento global, as espécies tropicais estão fazendo uma aposta bem mais arriscada (Pimm 2001).

\section{EFEITOS SOBRE A BIODIVERSIDADE BRASILEIRA}

O Neotrópico experienciará mudanças climáticas substanciais (IPCC 2007b). As previsões indicam que a porção tropical da América do Sul, incluindo a maior parte do território brasileiro, será a região mais afetada em termos de temperatura, com uma elevação de cerca de 2 a $6^{\circ} \mathrm{C}$ (Nobre et al. 2007). Em relação à precipitação, as regiões mais afetadas seriam a
Amazônia e o Nordeste do Brasil, com potencial para a ocorrência, respectivamente, de processos de savanização e arenização (Nobre et al. 2007). Os impactos dessas mudanças climáticas sobre a rica biodiversidade da região Neotropical já estão sendo sentidos e preocupam os profissionais em conservação da região (Ceballos et al. 2009). De fato, o primeiro caso registrado de extinção de espécie associada às mudanças climáticas ocorreu com um anfíbio endêmico da Costa Rica (Pounds et al. 1999). Em 2005, o Caribe vivenciou uma séria perturbação de seus ecossistemas de corais associada ao estresse térmico e fortes furacões (Wilkinson \& Souter 2008). No mesmo ano a Amazônia foi palco de um expressivo episódio de seca, gerando assombro geral diante da possível 'savanização' desse bioma de grande relevância internacional (Cox et al. 2004, CPTEC-INMET 2005, Hutyra et al. 2005, INPE 2005, Nobre et al. 2007, Salazar et al. 2007). Embora esse evento tenha sido relacionado às mudanças climáticas, Aziz Ab'Saber (geógrafo e professor emérito da Universidade de São Paulo) considera que o processo de savanização experimentado pela região amazônica nos últimos 25 anos é fruto da ação direta do homem, através das atividades de madeireiros, grileiros e, mais recentemente, produtores de soja (Brasil/CN/CME 2008). Menos divulgadas, mas igualmente espantosas, são as conseqüências das mudanças climáticas sobre ecossistemas andinos, ilustradas pela rápida retração do glaciar de Chacaltaya na Bolívia (Francou et al. 2003) e dos páramos de Los Nevados na Colômbia (Ruiz et al. 2008).

Se por um lado há ampla evidência, tanto registrada como projetada, de que o aquecimento está associado a mudanças na distribuição geográfica de espécies em regiões temperadas, por outro lado existem muito poucas tentativas de avaliar a sensibilidade dos trópicos às mudanças climáticas globais (Pounds et al.1999, Colwell et al. 2008, Svenning \& Condit 2008). De acordo com Colwell et al. (2008) os fracos gradientes latitudinais de temperatura encontrados nos trópicos criarão dificuldades para que as espécies acompanhem as condições climáticas convenientes através de migração pelas planícies. Por conseqüência, para espécies tropicais afetadas pelo aquecimento seria bem mais provável a ocorrência de mudanças de distribuição em direção a maiores elevações do que em direção a maiores latitudes (Bush \& Hooghiemstra 2005). Neste sentido, é exemplar o fato de que a primeira 
evidência publicada de mudanças contemporâneas em distribuição geográfica de espécies tropicais, registrada para alguns vertebrados da Costa Rica, corresponde a mudanças de elevação (Pounds et al. 2005). Empregando uma nova abordagem conceitual para analisar mudanças climáticas em ecossistemas tropicais, Colwell et al. (2008) propõem que nos trópicos o aquecimento global pode causar a extinção de três maneiras distintas: 'atrito biótico', 'lacunas na mudança de distribuição' e 'extinção de topo de montanha'. O primeiro processo incidiria sobre as planícies tropicais toda vez que o aquecimento forçasse as espécies para fora das planícies, ao mesmo tempo em que nenhuma fonte de espécies adaptadas a temperaturas mais altas estivesse disponível para compensar as perdas. O segundo modo incidiria também sobre as espécies de montanhas tropicais, ocorrendo em situações em que a distribuição altitudinal atual não apresentasse sobreposição com distribuições climaticamente favoráveis no futuro. $\mathrm{O}$ terceiro processo ocorreria no caso do aquecimento deslocar o espaço de condições climáticas favoráveis para além dos cumes das montanhas. Para avaliar o impacto potencial dos três modos de extinção sob condições de aquecimento projetado, Colwell et al. (2008) analisaram a distribuição de 2.000 espécies de plantas e insetos ao longo de uma transecção altitudinal de $2.900 \mathrm{~m}$, na Costa Rica. Os resultados indicaram que, com um aquecimento de $3,2^{\circ} \mathrm{C}, 53 \%$ das espécies estariam ameaçadas de extinção pelo 'atrito biótico' em planícies, $51 \%$ das espécies de montanha estariam ameaçadas pelas 'lacunas de mudança de distribuição' e apenas uma minoria enfrentaria a 'extinção de topo de montanha'. Segundo estes autores, as biotas de planícies tropicais podem enfrentar um nível de 'atrito biótico' sem paralelo em regiões temperadas, assim como uma boa parte das espécies tropicais em breve enfrentará o problema das 'lacunas' entre a distribuição altitudinal atual e futura.

Apesar de sua relevância e urgência, estudos sobre possíveis conseqüências das mudanças climáticas globais sobre a biodiversidade Neotropical são escassos. A clássica revisão de Root et al. (2003) sobre respostas das espécies às mudanças climáticas ilustra de maneira exemplar a falta de conhecimento sobre o tema nessa região. Baseados na metaanálise de 143 estudos com pelo menos 10 anos de duração, incluindo de moluscos a mamíferos e de gramíneas a árvores, os autores encontraram que, em $80 \%$ dos casos examinados, o parâmetro biológico medido apresentou uma resposta consistente com o aquecimento global esperado. A vasta maioria dos estudos examinados concentrava-se em organismos distribuídos na Europa e na América do Norte, com apenas um estudo contemplando a região Neotropical, realizado na América Central (Pounds et al. 1999). No que se refere à produção acadêmica sobre mudanças climáticas no Brasil, o número de artigos publicados nos últimos 20 anos analisados totaliza apenas 395 , representando pouco mais de $1 \%$ do total da produção mundial observada (Figura 3). Desde os artigos mais antigos encontrados, que datam de 1992, pode ser observado um crescimento moderado até 2006, seguido por um aumento aparentemente mais expressivo de produção em 2007. Contudo, cumpre ressaltar que, em termos de porcentagem anual em relação ao total global de artigos produzidos sobre mudanças climáticas, esta produção não exibe tendências de crescimento, apresentando apenas pequenas oscilações em torno de $1 \%$ ao longo de todo o período analisado.

No que concerne à produção acadêmica sobre a temática de mudanças climáticas e biodiversidade no Brasil, a situação é ainda mais crítica. O primeiro artigo encontrado na base ISI data de 1998, sendo que até 2007 a produção acumulou apenas 12 artigos (Figura 3). Existe, sobretudo, uma carência de estudos acerca dos efeitos de mudanças climáticas sobre organismos ocorrentes no Brasil. Uma busca por artigos publicados até 2007, que neste caso incluiu também periódicos nacionais e não indexados na base ISI, revelou apenas duas publicações. No primeiro estudo (Siqueira \& Peterson 2003), que analisou as distribuições de 162 espécies arbóreas do Cerrado projetadas em cenários futuros de mudanças climáticas, os resultados indicaram que todas as espécies experimentariam severa contração de mais de $50 \%$ em sua área de distribuição geográfica. Nas projeções em um cenário otimista, com aumento de até $2^{\circ} \mathrm{C}$ na temperatura média, 18 espécies desapareceriam por falta de área climaticamente favorável no futuro. Já no cenário mais realista, com aumento de até $4^{\circ} \mathrm{C}$, este número totaliza 56 espécies. O segundo estudo (Anciães \& Peterson 2006) também envolveu a projeção de distribuições potenciais em cenários futuros de mudança climática, com base na análise de 49 espécies de pássaros, entre 


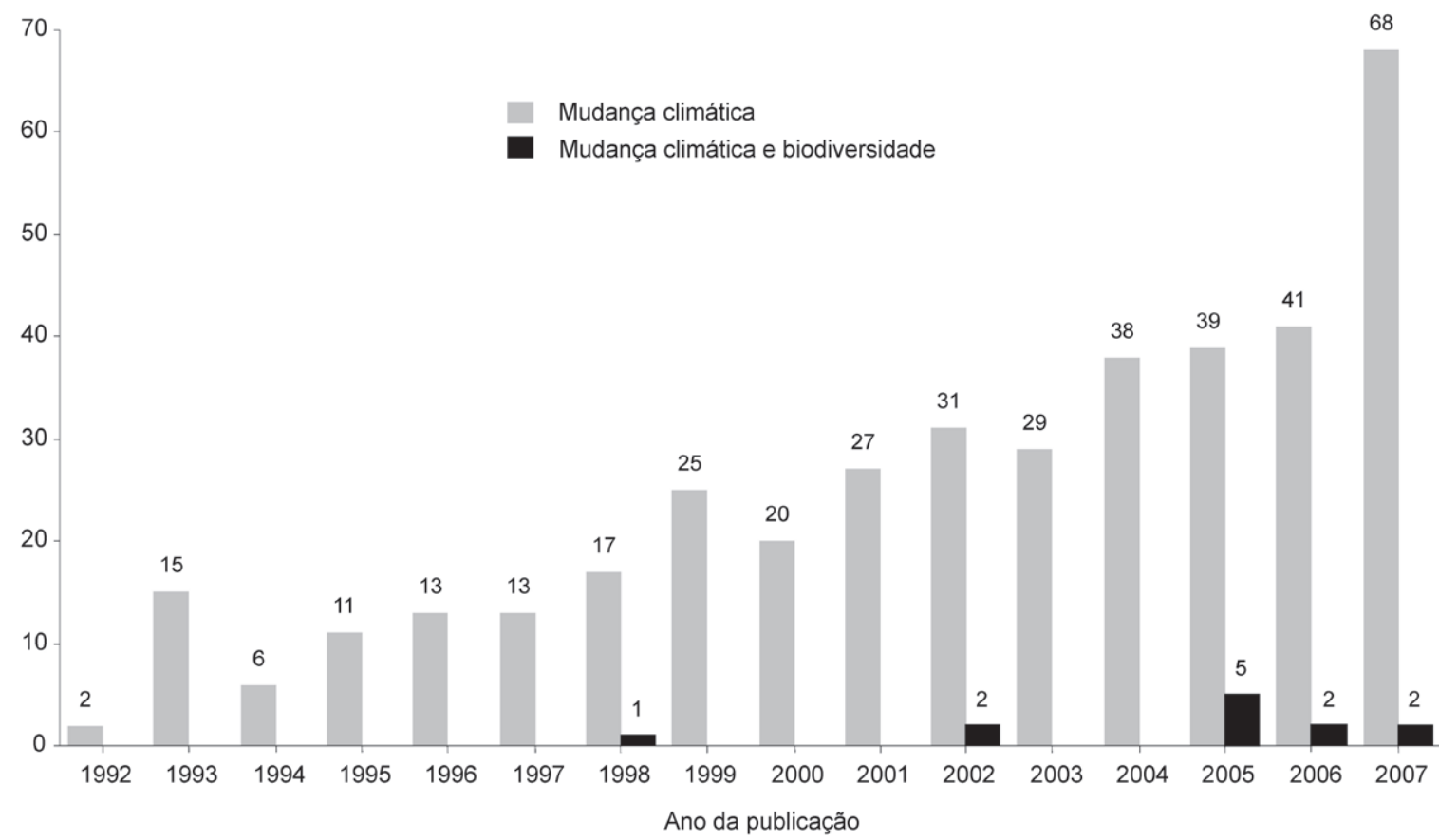

Figura 3. Tendência na publicação de artigos científicos relacionados ao temas 'mudança climática' $(n=395)$ e 'mudança climática associada à biodiversidade' $(n=12)$ no Brasil, ao longo dos últimos 20 anos. Os dados foram compilados a partir da base de dados Web of Science de publicações científicas do Thompson ISI (Thompson Institute for Scientific Information), através de uma busca por artigos publicados entre 1987 e 2007 , utilizando, respectivamente, os termos climat* change* mais brazil* e climat* change* mais biodiversity mais brazil* como palavras-chave. Figure 3. General trend of scientific articles published on the topic 'climate change' $(\underline{n}=395)$ and 'climate change and biodiversity' $(\underline{n}=12)$ over the last 20 years in Brazil. The number of articles is presented in white in the columns. Data were compiled from the Web of Science database in Thompson ISI (Thompson Institute for Scientific Information), based on a search for articles published between 1987-2007 with 'climat* chang*' plus 'brazil*', and 'climat* chang*'plus 'biodiversity' plus 'brazil*' as keywords.

tangarás e aves aparentadas da família Pipridae. Os resultados indicaram que, na ausência de dispersão, $20 \%$ das espécies estariam extintas pela ausência de área climaticamente favorável no futuro. Adicionalmente, as mudanças previstas na área de distribuição potencial, na configuração espacial dos hábitats favoráveis e na posição geográfica da distribuição das espécies foram maiores para aves habitantes de baixadas do que para aquelas de regiões montanhosas. Assim sendo, as conclusões de ambos os estudos sobre os impactos de mudanças climáticas em espécies brasileiras corroboram a idéia de que as espécies de planície também serão bastante afetadas pela falta de ambientes favoráveis no futuro, o que pode ameaçar seriamente a persistência da biodiversidade no Brasil.

O livro "A biologia e as mudanças climáticas no Brasil" (Buckeridge 2008), aparece como uma primeira iniciativa no sentido de sanar a enorme lacuna de conhecimento sobre as conseqüências das mudanças climáticas globais sobre a biodiversidade brasileira. O primeiro volume, lançado em dezembro de 2008, versa sobre vários temas nas dimensões atmosférica, biológica e humana das mudanças climáticas no Brasil. Na dimensão biológica é patente que as pesquisas sobre a flora parecem estar em estágio mais avançado do que os estudos relativos à fauna. Um exemplo é o capítulo de Buckeridge e colaboradores (2008), que apresenta os resultados de um estudo experimental sobre o estabelecimento de cinco espécies de Leguminosae arbóreas da Mata Atlântica, pertencentes a diferentes grupos funcionais, sob concentrações atuais e elevadas de $\mathrm{CO}_{2}$. $\mathrm{O}$ estudo chama a atenção para a importância das espécies pioneiras no processo de seqüestro de carbono. Essas espécies, que iniciam a sucessão ecológica, crescem rapidamente e seqüestram grandes quantidades de carbono por unidade de tempo, enquanto que as espécies de estágios sucessionais mais avançados crescem lentamente, seqüestrando menos carbono por unidade de tempo, embora realizem esse seqüestro por um tempo mais prolongado. Conseqüentemente, uma floresta tropical em regeneração seqüestraria carbono continuamente por um longo período. 
Além disso, o seqüestro de carbono é maior e mais consistente quando se executa o processo de sucessão em sistemas com múltiplas espécies em contraposição ao plantio de monoculturas. $\mathrm{O}$ estudo sugere ainda que as espécies intermediárias têm desempenho fisiológico melhor em relação às demais, de modo que essas espécies poderão ter maior probabilidade de adaptação às condições de elevado gás carbônico e pouca água previstas para o futuro.

Dentre os poucos estudos incluídos em Buckeridge (2008) sobre as respostas da fauna às mudanças climáticas destaca-se o de Haddad et al. (2008), que aborda os efeitos das mudanças climáticas na distribuição e declínio de anfíbios, um dos grupos mais sensíveis a alterações climáticas. A modelagem de re-distribuição em cenários de mudanças climáticas de espécies restritas às florestas ombrófilas montanas em diversos pontos da Mata Atlântica do Sudeste e, principalmente, do Sul do Brasil aponta para prováveis extirpações de populações de espécies de ampla distribuição, bem como para extinções globais de espécies endêmicas de distribuição restrita.

\section{OPORTUNIDADES PARA O BRASIL}

A realidade das mudanças climáticas globais exige uma resposta imediata tanto do governo quanto da sociedade civil. A publicação da revisão do Ministério do Meio Ambiente sobre os impactos das mudanças climáticas sobre a biodiversidade brasileira (Marengo 2007) e mais recentemente, do relatório final da Comissão Mista Especial Sobre Mudanças Climáticas do Congresso Nacional Brasileiro (Brasil/CN/CME 2008), são as primeiras iniciativas nesse sentido. É necessário desenvolver estratégias específicas de mitigação e adaptação às mudanças climáticas, sobretudo aquelas com possíveis conseqüências socioambientais e econômicas, tais como aumento da incidência de malária e dengue, perda de áreas agriculturáveis e deslocamento de populações humanas nas regiões litorâneas.

O governo brasileiro já criou um Comitê Interministerial sobre Mudança do Clima, formado por representantes de vários ministérios e por integrantes do Fórum Brasileiro de Mudanças Climáticas (FBMC), que ficou encarregado de elaborar um anteprojeto de lei a respeito da Política Nacional sobre Mudanças Climáticas (PNMC). O documento foi encaminhado ao Congresso Nacional em junho de $2008\left(\mathrm{PL} \mathrm{n}^{\circ}\right.$ 3.535, de 2008, na Câmara dos Deputados) e norteou a elaboração do Plano Nacional sobre Mudanças Climáticas, bem como outros planos, programas, projetos e ações relacionados (Brasil/CN/CME 2008). Em paralelo, já há uma série de proposições legislativas em tramitação no Congresso Nacional, ligadas às mudanças climáticas globais. São projetos de lei e propostas de emendas à constituição que dispõem, entre outros temas, sobre: (1) o sistema de vigilância das emissões de gases de efeito estufa; (2) realização do balanço de emissões de gases do efeitoestufa como parte do licenciamento ambiental; (3) incentivos fiscais àqueles que invistam em projetos de Mecanismo de Desenvolvimento Limpo; (4) inclusão dos impactos das mudanças climáticas entre as diretrizes para implementação do Sistema Nacional de Unidades de Conservação (ver Brasil/CN/CME 2008 para uma lista completa das proposições legislativas relevantes em tramitação).

O Plano Nacional sobre Mudança do Clima, apresentado em dezembro de 2008 pelo governo, em conjunto com o Projeto de Lei sobre a Política Nacional de Mudanças Climáticas, que está em tramitação no Congresso Nacional, representam as duas políticas macro para as mudanças climáticas no Brasil em nível federal. A primeira versão do Plano Nacional sobre Mudança do Clima apresentada pelo governo federal propôs metas voluntárias para a redução do desmatamento ilegal. A ser atingida até 2010, a meta estabelecida corresponde a uma diminuição do desmatamento em $40 \%$, em relação à média registrada entre os anos 1995 e 2005. Posteriormente, até 2017 espera-se obter uma redução do desmatamento de $30 \%$ a cada quatro anos. Outras medidas previstas pelo plano incluem crescimento anual de $11 \%$ no uso do etanol na matriz energética brasileira, aumento de $20 \%$ na co-geração de energia, bem como obter um maior número de árvores plantadas do que o de cortadas até 2015. O país pretende ainda reduzir em $10 \%$ o consumo de energia elétrica até 2030 , assim como até 2015 pretende iniciar a produção de células fotovoltaicas, gerar energia do lixo, estimular a construção de edifícios eficientes e alcançar uma taxa de reciclagem de resíduos de $20 \%$. Na fase de consulta pública (Brasil/CIMC, 2008) o plano foi criticado por diversas entidades da sociedade civil, que se articularam no sentido de propor contribuições a esse 
projeto (Observatório do Clima, 2008). A principal crítica refere-se à carência na apresentação de metas obrigatórias de redução de emissões de gases-estufa que pudessem ser quantificáveis, reportáveis e verificáveis.

Ao mesmo tempo, também no âmbito estadual estão surgindo iniciativas de políticas públicas relacionadas às mudanças climáticas. $\mathrm{O}$ estado do Amazonas, por exemplo, criou a Lei Estadual de Mudanças Climáticas ( $n^{\circ} 3.135$ ), primeira no país a tratar do tema, além de dispor do programa Bolsa Floresta, que contempla trabalhadores rurais inseridos em Reservas de Uso Sustentáveis do estado e que não praticam desmatamento (Viana \& Campos 2007). Para implementar a Lei 3.135 também foi criado neste estado o Centro Estadual de Mudanças Climáticas (CECLIMA), o primeiro centro governamental do Brasil especializado em articular políticas públicas sobre mudanças climáticas. Além do Amazonas, outras unidades da federação (por exemplo: Minas Gerais, Mato Grosso, Pernambuco, Piaú e São Paulo) atualmente possuem propostas de políticas estaduais que estão em discussão ou em fase de implementação.

Já em nível local tais iniciativas estão começando a surgir, cabendo destacar a posição pioneira do lançamento da Política Municipal de Mudança do Clima no Município de São Paulo, cujo Projeto de Lei (PL) foi encaminhado para votação pela Câmara de Vereadores em agosto de 2008 (Kassab 2008). O projeto apresenta propostas na área de construção, geraçãodeenergia, gerenciamentoderesíduos, licitação sustentável, saúde, transporte, entre outras. Neste PL fica estabelecida uma meta de redução de $30 \%$ das emissões de gases de efeito estufa do município até 2012. Para esta meta ser atingida ficam previstas a ampliação da oferta de transporte público, a restrição gradativa do acesso de veículos particulares ao centro da cidade, a melhoria no gerenciamento de resíduos e o desenvolvimento de projetos arquitetônicos com critérios de eficiência energética e sustentabilidade, entre outras diretrizes apontadas.

Do ponto de vista da geração de conhecimento, é preciso estimular a produção de estudos sobre os possíveis impactos das mudanças climáticas para a biodiversidade brasileira. Essa é uma tarefa que cabe não apenas aos órgãos de fomento à pesquisa, mas também aos próprios pesquisadores. Os estudos de longa duração são sem dúvida necessários, mas sua implementação é caracteristicamente onerosa. Uma alternativa bastante viável e que tem sido sub-explorada são as modelagens ecológicas que prevêm a re-distruibição de espécies em cenários de mudanças climáticas (por exemplo: Siqueira \& Peterson 2003, Anciães \& Peterson 2006, Haddad et al. 2008). É uma abordagem rápida, que exige dados muitas vezes disponíveis na Internet e para a qual já existem pacotes analíticos acessíveis (Elith et al. 2006). A despeito das limitações apresentadas por este tipo de análise (Pearson \& Dawson 2003, Hampe 2004, Thuiller 2004, Araújo \& Pearson 2005, Ibáñez et al. 2006, Akçakaya et al. 2006, Araújo \& Luoto 2007, Botkin et al. 2007, Thuiller et al. 2008), existe consenso de que, para avaliar grandes conjuntos de espécies, as abordagens de modelagem provêem os melhores métodos atuais para predizer mudanças induzidas pelo clima sobre a distribuição de espécies. Deste modo, estas análises têm o potencial de gerar alguma luz emuma área que está absolutamente carente de conhecimento, como discutido anteriormente.

O Brasil, no entanto, não deve se concentrar apenas em estratégias de 'adaptação', pois existe grande potencial para ações de 'mitigação' das mudanças climáticas em andamento. O país é responsável por uma porção substancial da emissão global de gases de efeito estufa. Embora nossa emissão por queima de combustíveis fósseis seja modesta, sobretudo devido à matriz energética limpa do país, a emissão nacional derivada do desmatamento é enorme. A média anual de $17.900 \mathrm{~km}^{2}$ em área de floresta que é desmatada na Amazônia desde 1988 (INPE 2008), combinada com uma emissão média de $200 \mathrm{tC} / \mathrm{ha}$ na conversão de florestas maduras (Luyssaert et al. 2008), equivale a uma emissão de aproximadamente 360 milhões de toneladas de carbono por ano. Essa estimativa coloca o Brasil entre os países que mais emitem gases de efeito estufa no mundo, ao lado dos Estados Unidos, China e Rússia, mesmo sem contabilizar as emissões por queima de combustíveis fósseis ou por desmatamento fora da Amazônia. Nas suas taxas atuais, o desmatamento no Brasil equivale a $40 \%$ do alvo de redução anual para países desenvolvidos dentro do Protocolo de Kyoto (Santilli et al. 2005). É importante notar que a maior parte do PIB da Amazônia está associada a atividades econômicas que geram pouco desmatamento, como 
por exemplo, a exploração mineral. As grandes vilãs do desmatamento, ou seja, as atividades ligadas à criação de gado, produção de soja e de madeira, representam menos de $1 \%$ do PIB brasileiro (C. Nobre, comunicação pessoal). Portanto, a idéia de que a redução do desmatamento na Amazônia representaria um revés econômico para o Brasil, ou mesmo para a região norte, não se sustenta.

O Protocolo de Kyoto prevê a redução nas emissões de países desenvolvidos através do investimento em projetos de reflorestamento e florestamento (conversão de área não-florestada há mais de 50 anos em floresta) executados em países em desenvolvimento, como parte do Mecanismo de Desenvolvimento Limpo (MDL). Até hoje, somente estes tipos de atividades de 'Uso da Terra, Mudança no Uso da Terra e Florestas' (LULUCF, na sigla em inglês) que visam remover gás carbônico da atmosfera permanecem elegíveis dentro do MDL. Países com altas taxas de desmatamento, no entanto, não têm qualquer incentivo dentro do protocolo para reduzir ou evitar emissões associadas ao desmatamento. Em 2005, a Convenção-Quadro das Nações Unidas sobre Mudanças Climáticas (UNFCCC, na sigla em inglês) lançou uma iniciativa para avaliar a possibilidade de incorporar a redução de emissão por desmatamento em países em desenvolvimento nos mecanismos do Protocolo de Kyoto (UN 2005). Espera-se que até 2009 um mecanismo de Redução das Emissões do Desmatamento e da Degradação (REDD) possa ser incorporado no acordo de clima que sucederá o Protocolo de Kyoto após 2012 (Pinto et al. 2008).

Embora o Brasil tenha sido um dos propositores do MDL em 1997, o país sempre se opôs à inclusão de florestas naturais nesse dispositivo, baseando-se em razões econômicas e em uma antiquada doutrina de soberania nacional (Fearnside 2001, Leite 2007, Fearnside no prelo a). A razão econômica baseia-se na idéia de que a enorme extensão de florestas que ainda resta no mundo - incluindo as tropicais e principalmente as boreais - faria com que o preço dos créditos de carbono despencasse no mercado mundial. Essa é a posição mantida pelas delegações brasileiras, postura que muitas vezes contrasta com o que defendem alguns ambientalistas no Brasil, em especial aqueles ligados à preservação das florestas. Como alternativa, sob a forma da criação de fundos internacionais surgem várias propostas de financiamento da preservação da floresta em pé, que estariam desvinculadas do Protocolo de Kyoto e, portanto, sem nenhuma relação com créditos de carbono (V.C.M. Baraviera, comunicação pessoal).

Segundo a Comissão Mista Especial Sobre Mudanças Climáticas do Congresso Nacional: "Poderse-ia, segundo especialistas, criar instrumentos econômicos para custear o combate ao desmatamento, como estabelecer tributos sobre o que é produzido no âmbito da Floresta Amazônica e aplicar essa arrecadação em ações que ajudem a preservá-la. Poderiam também ser buscados recursos no plano internacional. Seria, contudo, mais fácil arrecadar recursos estrangeiros se o Brasil assumisse metas de diminuição do desmatamento perante a comunidade internacional. Outra proposta é a criação de um mecanismo novo, no âmbito da Convenção do Clima, complementar ao Protocolo de Kyoto, para contemplar o desmatamento evitado. Além disso, propõe-se que sejam estabelecidos instrumentos tributários no comércio internacional, privilegiando produtos da Amazônia cujas cadeias produtivas contribuam para a conservação ambiental e para o desenvolvimento sustentável. Uma terceira proposta consiste no estabelecimento de uma estratégia de redução compensada do desmatamento. Países em desenvolvimento, que se dispusessem e conseguissem promover reduções das suas emissões nacionais oriundas de desmatamento, receberiam compensação financeira internacional correspondente às emissões evitadas, tendo como referência a taxa média de desmatamento anual e um valor médio do carbono no mercado internacional. Essa compensação poderia ocorrer por meio de um fundo internacional ou por meio do mercado de carbono, que já existe. Reduzir a atual taxa de desmatamento da Amazônia em $10 \%$, por exemplo, segundo estimativa do Instituto, geraria uma receita de aproximadamente 500 milhões de dólares por ano" (BRASIL/CN/CME 2008).

O Ministério do Meio Ambiente já está caminhando nesse sentido e finalmente conseguiu lançar a proposta de criação de um fundo voluntário em que países desenvolvidos depositariam recursos para apoiar projetos que implicassem em redução de desmatamento em países em desenvolvimento (Leite 2007). A incorporação de compensação por 'desmatamento evitado' no Protocolo de Kyoto é apoiada também por diversos pesquisadores no Brasil (por exemplo: Santilli et al. 2005, Fonseca et al. 2007, Young et al. 2007, Fearnside no prelo a). 
O ‘desmatamento evitado’ é, sem dúvida, um mecanismo com grande potencial para reduzir a emissão global de gases de efeito estufa e estimular uma participação mais efetiva de países em desenvolvimento no Protocolo de Kyoto (Santilli et al. 2005, Gullison et al. 2007, Malhi et al. 2008, Fearnside no prelo b). Muito além de proteger o clima, a redução de desmatamento de florestas tropicais tem o potencial de eliminar muitos dos impactos negativos que podem comprometer a habilidade dos países tropicais para desenvolver sustentabilidade, tais como a redução da pluviosidade, a erosão da biodiversidade, a deterioração da saúde humana oriunda da poluição pela queima de biomassa e a perda não intencional de florestas produtivas (Chomitz et al. 2006, Gullison et al.2007). A compensação por 'desmatamento evitado' poderia auxiliar a resolver potenciais conflitos entre as Convenções do Clima e da Biodiversidade (Santili et al. 2005), reforçando a desejada e necessária sinergia que deve ser estabelecida entre as duas convenções. Além disso, esse mecanismo tem a possibilidade de gerar divisas para o Brasil e reduzir as embaraçosas taxas de desmatamento do país.

O Brasil tem grande poder de negociação dentro da UNFCCC (Fearnside 2001) e o apoio à incorporação de compensação por 'desmatamento evitado' seria sem dúvida a maior contribuição que o país poderia fazer para reverter o quadro atual de mudanças climáticas. Por fim, cabe destacar que conservar a biodiversidade em um mundo de mudanças climáticas será um grande desafio, que necessitará tanto de medidas de adaptação, para aperfeiçoar as estratégias de conservação, quanto de ações de mitigação, para estabilizar os níveis atmosféricos de gases de efeito estufa (Hannah et al. 2007). Segundo Jetz et al. (2007), para minimizar as extinções globais projetadas, será necessário combinar a manutenção de uma rede de áreas protegidas bastante expandida nos trópicos e a adoção de objetivos mais ambiciosos para reduzir as mudanças climáticas. Neste sentido, e acompanhando a abordagem de 'desmatamento evitado', as áreas protegidas podem ser uma importante estratégia de conservação no cenário de mudanças climáticas globais (Hannah et al. 2007, Soares-Filho et al. 2008, Fearnside no prelo a, b). É importante que a comunidade científica brasileira ligada à conservação participe ativamente do debate sobre o 'desmatamento evitado' e as mudanças climáticas, pois as conseqüências para a conservação da biodiversidade são enormes.
AGRADECIMENTOS: Agradecemos Marcos Buckeridge e Alexandre Diniz-Filho pela revisão e sugestões ao manuscrito e Carlos Eduardo V. Grelle pela possibilidade de participar deste número especial da Oecologia Brasiliensis. Agradecemos também a Verônica de Carvalho Maia Baraviera pela disponibilização de material produzido pelo Congresso Nacional brasileiro e Fabiana dos Santos e Souza pela disponibilização de material relativo às políticas públicas no estado do Amazonas. Este estudo contou com apoio do CNPq através de uma bolsa de Pós-doutorado para MMV e de Produtividade em Pesquisa (com grant associado) para MASA (processo n.302718/2003-6), bem como da CAPES e do Programa Nacional de Pós-Doutorado (PNPD) através de uma bolsa de Pós-doutorado para MLL.

\section{REFERÊNCIAS}

AKÇAKAYA, H.R.; BUTCHART, S.H.M.; MACE, G.M.; STUART, S.N. \& HILTON-TAYLOR, C. 2006. Use and misuse of the IUCN Red List Criteria in projecting climate change impacts on biodiversity. Global Change Biology, 12: 2037-2043.

ANCIÃES, M. \& PETERSON, A.T. 2006. Climate change effects on neotropical manakin diversity based on ecological niche modeling. The Condor, 108: 778-791.

ARAÚJO, M.B. \& LUOTO, M. 2007. The importance of biotic interactions for modelling species distributions under climate change. Global Ecology and Biogeography, 16: 743-753.

ARAÚJO, M.B. \& PEARSON, R.G. 2005. Equilibrium of species' distributions with climate. Ecography, 28: 693-695.

AUSTIN, G.E. \& REHFISCH, M.M. 2005. Shifting nonbreeding distributions of migratory fauna in relation to climatic change. Global Change Biology, 11: 31-38.

BAKKENES, M.; ALKEMADE, J.R.M.; IHLE, F.; LEEMANS, R. \& LATOUR, J.B. 2002. Assessing effects of forecasted climate change on the diversity and distribution of European higher plants for 2050. Global Change Biology, 8: 390-407.

BOTKIN, D.B.; SAXE, H.; ARAÚJO, M.B.; BETTS, R.; BRADSHAW, R.H.W.; CEDHAGEN, T.; CHESSON, P.; DAWSON, T.P.; ETTERSON, J.R.; FAITH, D.P.; FERRIER, S.; GUISAN, A.; HANSEN, A.S.; HILBERT, D.W.; LOEHLE, C.; MARGULES, C.; NEW, M.; SOBEL, M.J. \& STOCKWELL, D.R.B. 2007. Forecasting the Effects of Global Warming on Biodiversity. BioScience, 57(3): 227-236.

BRASIL/CIMC. 2008. Plano Nacional sobre Mudança do Clima - Versão para consulta pública. Comitê Interministerial Sobre Mudança do Clima, Brasília. 154p. http://www.mma.gov.br/ estruturas/ascom_boletins/_arquivos/83_26092008095928.pdf (acesso em 13/06/2009).

BRASIL/CN/CME. 2008. Comissão Mista Especial sobre Mudanças Climáticas (CME): Relatório Final. Congresso 
Nacional, Brasília. 246p. http://www2.senado.gov.br/bdsf/item/ id/98732 (acesso em 18/11/2008).

BUCKERIDGE, M.S.; AIDAR, M.P.M.; MARTINEZ, C.A. \& SILVA, E.A. 2008. Respostas de plantas às mudanças climáticas globais. Pp. 77-91. In: M.S. Buckeridge (Ed.) A biologia e as mudanças climáticas no Brasil. RiMa Editora, São Carlos. 316p.

BUCKERIDGE, M.S. 2008 (org). A biologia e as mudanças climáticas no Brasil. RiMa Editora, São Carlos. 316p.

BUSH, M.B. \& HOOGHIEMSTRA, H. 2005. Tropical biotic responses to climate change. Pp. 125-137. In: T.E. Lovejoy \& L. Hannah (eds.). Climate Change and Biodiversity. Yale University Press, New Haven \& London. 440p.

CEBAllos, G.; VALE, M.M.; BONACIC, C.; CALVO, J.; LIST, R.; BYNUM, N.; MEDELLÍN, R.A.; SIMONETTI, J.A. \& RODRÍGUEZ, J.P. 2009. Conservation Challenges for the Austral and Neotropical America Section. Conservation Biology, 23: 811-817.

CHOMITZ, K.; BUYS, P.; De LUCA, G.; THOMAS, T.S. \& WERTZ-KANOUNNIKOF, S. 2006. At Loggerheads? Agricultural Expansion, Poverty Reduction, and Environment in Tropical Forests. World Bank Publications, Washington. 304p.

COLWELL, R.K.; BREHM, G.; CARDELÚS, C.L.; GILMAN, A.C. \& LONGINO, J.T. 2008. Global warming, elevational range shifts, and lowland biotic attrition in the wet tropics. Science, 322: $258-261$.

COX P.M.; BETTS, R.A.; COLLINS, M.; HARRIS, C.; HUNTINGFORD, C. \& JONES, C.D. 2004. Amazon dieback under climate-carbon cycle projections for the 21 st century. Theoretical and Applied Climatology, 78: 137-156.

CPTEC-INMET. 2005. Seca da Amazônia em 2005. Nota ao público em geral elaborada pelo Centro de Previsão de Tempo e Estudos Climáticos (CPTEC) em conjunto com o Instituto Nacional de Meteorologia (INMET). Agência CT, Ministério da Ciência e Tecnologia, Brasília. 3p.

CRICK, H.Q.P.; DUDLEY, C.; GLUE, D.E. \& THOMSON, D.L. 1997. UK birds are laying eggs earlier. Nature, 388: 526-526.

DAVIS, M.B. \& SHAW, R.G. 2001. Range shift and adaptive responses to Quaternary climate change. Science, 292: 673-679.

DINIZ-FILHO, J.A.F. \& BINI, L.M. 2008. Macroecology, global change and the shadow of forgotten ancestors. Global Ecology and Biogeography, 17(1): 11-17.

ELITH, J.; GRAHAM, C.H.; ANDERSON, R.P.; DUDÍK, M.; FERRIER, S.; GUISAN, A.; HIJMANS, R.J.; HUETTMANN, F.;
LEATHWICH, J.R.; LEHMANN, A.; LI, J.; LOHMANN, L.G.; LOISELLE, B.A.; MANION, G.; MORITZ, C.; NAKAMURA, M.; NAKAZAWA, Y.; OVERTON, J.M.; PETERSON, A.T.; PHILLIPS, S.J.; RICHARDSON, K.; SCACHETTI-PEREIRA, R.; SCHAPIRE, R.E.; SOBERÓN, J.; WILLIAMS, S.; WISZ, M.S. \& ZIMMERMANN, N.E. 2006. Novel methods improve prediction of species' distribution from occurrence data. Ecography, 29: 129-151.

ERASMUS, B.F.N.; VAN JAARSVELD, A.S.; CHOWN, S.L.; KSHATRIYA, M. \& WESSELS, K.J. 2002. Vulnerability of South African animal taxa to climate change. Global Change Biology, 8: 679-693.

FEARNSIDE, P.M. (a). No prelo. Chapter 48: Tropical Forests in Mitigating Climate Change. In: S. Schneider, A. Rosencranz \& M. Mastrandrea (eds.). Climate Change Science and Policy. Island Press, Covelo.

FEARNSIDE, P.M. (b). No prelo. O valor de áreas protegidas em evitar mudança climática na Amazônia. In: A.K. Albernaz (ed.) Atualização das Áreas Prioritárias para a Conservação, Uso Sustentável e Repartição dos Benefícios da BiodiversidadeBioma Amazônia. Ministério do Meio Ambiente, Brasília.

FEARNSIDE, P.M. 2001. Saving tropical forests as a global warming countermeasure: An issue that divides the environmental movement. Ecological Economics, 39(2): 167-184.

FEARNSIDE, P.M. 2008. As mudanças climáticas globais e a floresta amazônica. Pp.131-150. In: M.S. Buckeridge (Ed.) A biologia e as mudanças climáticas no Brasil. RiMa Editora, São Carlos. 316p.

FISCHLIN, A.; MIDGLEY, G.F.; PRICE, J.T.; LEEMANS, R.; GOPAL, B.; TURLEY, C.; ROUNSEVELL, M.D.A.; DUBE, O.P.; TARAZONA, J. \& VELICHKO, A.A. 2007. Ecosystems, their properties, goods, and services. Pp. 211-272. In: M.L. Parry, O.F. Canziani, J.P. Palutikof, P.J. van der Linden \& C.E. Hanson (eds.). Climate change 2007: impacts, adaptation and vulnerability. Contribution of Working Group II to the fourth assessment report of the Intergovernmental Panel on Climate Change. Cambridge University Press, Cambridge. 976p.

FODEN, W.; MIDGLEY, G.F.; HUGHES, G.O.; BOND, W.J.; THUILLER, W.; HOFFMAN, M.T.; KALEME, P.; UNDERHILL, L.G.; REBELO, A.G. \& HANNAH, L. 2007. A changing climate is eroding the geographical range of the Namib Desert tree Aloe through population declines and dispersal lags. Diversity and Distributions, 13: 645-653.

FONSECA, G.A.B.; RODRIGUEZ, C.M.; MIDGLEY, G.; BUSCH, J.; HANNAH, L. \& MITTERMEIER, R.A. 2007. No Forest Left Behind. Plos Biology, 5(8): 1645-1646. 
FRANCO,A.M.A.;HILL, J.K.; KITSCHKE, C.; COLLINGHAM, Y.C.; ROY, D.B.; FOX, R.; HUNTLEY, B. \& THOMAS, C.D. 2006. Impacts of climate warming and habitat loss on extinctions at species' low-latitude range boundaries. Global Change Biology, 12(8): 1545-1553.

FRANCOU, B.; VUILLE, M.; WAGNON, P.; MENDOZA, J. \& SICART. J. 2003. Tropical climate change recorded by a glacier in the central Andes during the last decades of the twentieth century: Chacaltaya, Bolivia, $16^{\circ} \mathrm{S}$. Journal of Geophysical Research, 108: 4154-4163.

GRAHAM，R.W.; LUNDELIUS，E.L.JR; GRAHAM， M.A.; SCHROEDER, E.K.; TOOMEY, R.S.III; ANDERSON, E.; BARNOSKY, A.D.; BURNS, J.A.; CHURCHER, C.S.; GRAYSON，D.K.; GUTHRIE，R.D.; HARINGTON，C.R.; JEFFERSON, G.T.; MARTIN, L.D.; MCDONALD, H.G.; MORLAN, R.E.; SEMKEN, H.A.JR; WEBB, S.D.; WERDELIN, L. \& WILSON, M.C. 1996. Spatial Response of Mammals to Late Quaternary Environmental Fluctuations. Science, 272:1601-1606.

GULLISON, R.E.; FRUMHOFF, P.C.; CANADELL, J.G.; FIELD, C.B.; NEPSTAD, D.C.; HAYHOE, K.; AVISSAR, R.; CURRAN, L.M.; FRIEDLINGSTEIN, P.; JONES, C.D. \& NOBRE, C. 2007. Tropical forests and climate policy: new science underscores the value of a climate policy initiative to reduce emissions from tropical deforestation. Science, 316: $985-986$.

HADDAD, C.F.B.; GIOVANELLI, J.G.R. \& ALEXANDRINO, J. 2008. O aquecimento global e seus efeitos na distribuição e declínio dos anfíbios. Pp. 195-206. In: M.S. Buckeridge (Ed.) A biologia e as mudanças climáticas no Brasil. RiMa Editora, São Carlos. 316p.

HAMPE, A. 2004. Bioclimate envelope models: what they detect and what they hide. Global Ecology and Biogeography, 13: 469-471.

HANNAH, L.; MIDGLEY, G.F.; ANDELMAN, S.; ARAÚJO, M.B.; HUGHES, G.O.; MARTINEZ-MEYER, E.; PEARSON, R.G. \& WILLIAMS, P.H. 2007. Protected area needs in a changing climate. Frontiers in Ecology and the Environment, 5: 131-138.

HICKLING, R.; ROY, D.B.; HILL, J.K. \& THOMAS, C.D. 2005. A northward shift of range margins in British Odonata. Global Change Biology, 11: 502-506.

HIGGINS, S.I.; RICHARDSON, D.M. \& COWLING, R.M. 2000. Using a dynamic landscape model for planning the management of alien plant invasions. Ecological Applications, 10: $1833-1848$
HOLT, R.D. 1990. The microevolutionary consequences of climate change. Trends in Ecology and Evolution, 5: 311-315.

HUGHES, L. 2000. Biological consequences of global warming: is the signal already apparent? Trends in Ecology and Evolution, 15: $56-61$

HUTYRA, L.R.; MUNGER, J.W.; NOBRE, C.A.; SALESKA, S.R.; VIEIRA, S.A. \& WOFSY, S.C. 2005. Climatic variability and vegetation vulnerability in Amazônia. Geophysical Research Letters, 32(24): L24712.1-L24712.4.

IBÁÑEZ，I.; CLARK，J.S.; DIETZE，M.C.; FEELEY，K.; HERSH, M.; LADEAU, S.; MCBRIDE, A.; WELCH, N.E. \& WOLOSIN, M.S. 2006. Predicting biodiversity change: outside the climate envelope, beyond the species-area curve. Ecology, 87: 1896-1906.

INPE (Instituto Nacional de Pesquisas Espaciais). 2005. Seca na Amazônia em 2005 http://www.inpe.br/noticias/noticia. php?Cod_Noticia=492 (acesso em 15/11/2008).

INPE (Instituto Nacional de Pesquisas Espaciais). 2008. Projeto Prodes: Monitoramento da Floresta Amazônica Brasileira por Satélite. Estimativas Anuais desde 1988 até 2007. http:// www.obt.inpe.br/prodes/prodes_1988_2007.htm (acesso em 28/10/2008).

IPCC (Intergovernmental Panel on Climate Change). 2007a. Climate Change 2007: Synthesis Report. Cambridge University Press, Cambridge. 133p.

IPCC (Intergovernmental Panel on Climate Change). 2007b. Latin America. Pp 583-615. In: Intergovernmental Panel on Climate Change, Climate Change 2007: Impacts, Adaptation and Vulnerability. Cambridge University Press, Cambridge. 976p.

JETZ, W.; WILCOVE, D.S. \& DOBSON, A.P. 2007. Projected Impacts of Climate and Land-Use Change on the Global Diversity of Birds. PLoS Biology, 5(6): 1211-1219.

KASSAB, G. Projeto de Lei $n^{\circ}$ 530/2008 de 14/08/2008 - Institui A Política Municipal de Mudança do Clima no Município de São Paulo. http://www.camara.sp.gov.br/ (acesso em 13/06/2009).

KERR, J. \& PACKER, L. 1998. The impact of climate change on mammal diversity in Canada. Environmental Monitoring and Assessment, 49: 263-270.

LAVERGNE, S.; MOLINA, J. \& DEBUSSCHE, M. 2006. Fingerprints of environmental change on the rare Mediterranean flora: a 115-year study. Global Change Biology, 12: 1466-1478.

LEITE, M. 2007. Um novo clima no Brasil. Ciência \& Ambiente 34: 5-9. 
LEVINSKY, I.; SKOV, F.; SVENNING, J.C. \& RAHBEK, C. 2007. Potential impacts of climate change on the distributions and diversity patterns of European mammals. Biodiversity Conservation, 16: 3803-3816.

LOVEJOY, T.E. \& HANNAH, L. (eds.) 2005. Climate Change and Biodiversity. Yale University Press, New Haven \& London. 440p.

LUYSSAERT, S.; SCHULZE, E.D.; BÖRNER, A.; KNOHL, A.; HESSENMÖLLER, D.; LAW, B.E.; CIAIS, P. \& GRACE, J. 2008. Old-growth forests as global carbon sinks. Nature, 455: 213-215.

MALCOLM, J.R.; LIU, C.; NEILSON, R.P.; HANSEN, L. \& HANNAH, L. 2006. Global Warming and Extinctions of Endemic Species from Biodiversity Hotspots. Conservation Biology, 20(2): 538-548.

MALCOLM, J.R.; MARKHAM，A.; NEILSON， R.P. \& GARACI, M. 2002. Estimated migration rates under scenarios of global climate change. Journal of Biogeography, 29: 835-849.

MALHI, Y.; ROBERTS, T.; BETTS, R.A.; KILLEEN, T.J.; LI, W. \& NOBRE, C.A. 2008. Climate Change, Deforestation, and the Fate of the Amazon. Science, 319: 169-172.

MARENGO, J.A. 2007. Mudanças Climáticas Globais e seus Efeitos sobre a Biodiversidade: caracterização do clima atual e definição das alterações climátcas para o território brasileiro ao longo do século XXI. $2^{\mathrm{a}}$. Edição. Ministério do Meio Ambiente, Brasília. 212p.

MARTÍNEZ-MEYER, E.; TOWNSEND, A.T. \& HARGROVE, W.W. 2004. Ecological niches as stable distributional constraints on mammal species, with implications for Pleistocene extinctions and climate change projections for biodiversity. Global Ecology and Biogeography, 13: 305-314.

MCCARTY, J.P. 2001. Ecological consequences of recent climate change. Conservation Biology, 15: 320-331.

MCKENNEY， D.W.; PEDLAR， J.H.; LAWRENCE， K.; CAMPBELL, K. \& HUTCHINSON, M.F. 2007. Potential Impacts of Climate Change on the Distribution of North American Trees. BioScience, 57(11): 939-948.

MEA (Millennium Ecosystem Assessment). 2005. Ecosystems and Human Well-being: Biodiversity Synthesis. World Resources Institute, Washington. 86p.

MEEHL, G.A.; STOCKER, T.E.; COLLINS, W.D.; FRIEDLINGSTEIN, P.; GAYE, A.T.; GREGORY, J.M.; KITOH, A.; KNUTTI, R.; MURPHY, J.M.; NODA, A.; RAPER, S.C.B.; WATTERSON, G.; WEAVER J. \& ZHAO, Z.C. 2007:
Global Climate Projections. Pp. 747-845. In: S. Solomon, D. Qin, M. Manning, Z. Chen, M. Marquis, K.B. Averyt, M. Tignor \& H.L. Miller (eds.). Climate Change 2007: The Physical Science Basis. Cambridge University Press, Cambridge. 996p.

MENZEL, A. \& FABIAN, P. 1999. Growing season extended in Europe. Nature, 397: 659.

MEYNECKE, J.O. 2004. Effects of global climate change on geographic distributions of vertebrates in North Queensland. Ecological Modelling, 174: 347-357.

MIDGLEY, G.F.; THUILLER, W. \& HIGGINS, S.I. 2007. Plant species migration as a key uncertainty in predicting future impacts of climate change on ecosystems: progress and challenges. Pp. 129-137. In: J. Canadell, D.E. Pataki \& L.F. Pitelka (eds.). Terrestrial ecosystems in a changing world. Springer, Berlim. 336p.

MØLLER, A.P.; FLENSTED-JENSEN, E. \& MARDAL, W. 2006. Dispersal and climate change: a case study of the Arctic tern Sterna paradisaea. Global Change Biology, 12: 2005-2013.

MORITZ, C.; PATTON, J.L.; CONROY, C.J.; PARRA, J.L.; WHITE, G.C. \& BEISSINGER, S.R. 2008. Impact of a Century of Climate Change on Small-Mammal Communities in Yosemite National Park, USA. Science, 322: 261-264.

NOBRE, C.A.; LAPOLA, D.; SAMPAIO, G.; SALAZAR, L.F.; CARDOSO, M. \& OYAMA, M. 2007. Mudanças Climáticas Globais e Possíveis Alterações nos Biomas da América do Sul. Relatório $n^{\circ}$ 6. Ministério do Meio Ambiente - MMA, Secretaria de Biodiversidade e Florestas - SBF, Diretoria de Conservação da Biodiversidade - DCBIO. 25p.

OBSERVATÓRIO DO CLIMA. 2008. Elementos para Formulação de um Marco Regulatório em Mudanças Climáticas no Brasil: Contribuições da Sociedade Civil. Observatório do Clima - Rede Brasileira de ONGs e Movimentos Sociais em Mudanças Climáticas, Brasília. 103p. http://intranet.gvces.com. br/arquivos/Justificativa_PL_OC_r.pdf (acesso em 13/06/2009).

OPDAM, P. \& WASCHER, D. 2004. Climate change meets habitat fragmentation: linking landscape and biogeographical scale levels in research and conservation. Biological Conservation, 117: 285-297.

PARMESAN, C. \& YOHE, G. 2003. A globally coherent fingerprint of climate change impacts across natural systems. Nature, 421: 37-42.

PARMESAN, C. 2006. Ecological and evolutionary responses to recent climate change. Annual Review of Ecology, Evolution and Systematics, 37: 637-669. 
PAULI, H.; GOTTFRIED, M.; REITER, K.; KLETTNER, C. \& GRABHERR, G. 2006. Signals of range expansions and contractions of vascular plants in the high Alps: observations (1994-2004) at the GLORIA master site Schrankogel, Tyrol, Austria. Global Change Biology, 13: 147-156.

PEARSON, R.G. \& DAWSON, T.E. 2003. Predicting the impacts of climate change on the distribution of species: are bioclimate envelope models useful? Global Ecology and Biogeography, 12: 361-372.

PETERSON, A.T.; ORTEGA-HUERTA, M.A.; BARTLEY, J.; SÁNCHEZ-CORDERO, V.; SOBERÓN, J.; BUDDEMEIER, R.H. \& STOCKWELL, D.R.B. 2002. Future projections for Mexican faunas under global climate change scenarios. Nature, 416: 626-629.

PETERSON, A.T.; SÁNCHEZ-CORDERO, V.; SOBERÓN, J.; BARTLEY, J.; BUDDEMEIER, R.W. \& NAVARROSIGÜENZA, A.G. 2001. Effects of global climate change on geographic distributions of Mexican Cracidae. Ecological Modelling, 144: 21-30

PIMM S.L. 2001. Intrepeneurial Insects. Nature, 411: 531-532.

PINTO, E.P.P.; MOUTINHO, P. \& RODRIGUES, L. 2008. Perguntas e respostas sobre aquecimento global. $3^{\mathrm{a}}$ ed. IPAM Instituto de Pesquisa Ambiental da Amazônia, Belém. 52p.

POUNDS, A.J.; BUSTAMANTE, M.R.; COLOMA, L.A.; CONSUEGRA, J.A.; FOGDEN, M.P.L.; FOSTER, P.N.; LA MARCA, E.; MASTERS, K.L.; MERINO-VITERI, A.; PUSCHENDORF, R.; RON, S.R.; SANCHEZ-AZOFEIFA, G.A.; STILL, C.J. \& YOUNG, B.E. 2006. Widespread amphibian extinctions from epidemic disease driven by global warming. Nature, 439: 161-167.

POUNDS, J.A.; FOGDEN, L.P. \& CAMPBELL, J.H. 1999. Biological response to climate change on a tropical mountain. Nature, 398: 611-615.

POUNDS, J.A.; FOGDEN, M.P.L. \& MASTERS, K.L. 2005. Response of communities to climate change in a highland tropical forest. Pp. 70-74. In: T.E. Lovejoy \& L. Hannah (eds.). Climate Change and Biodiversity. Yale University Press, New Haven \& London. 440p.

ROOT, T.L.; MACMYNOWSKI，D.P.; MASTRANDREA, M.D. \& SCHNEIDER, S.H. 2005. Human-modified temperatures induce species changes: joint attribution. Proceeding of the National Academy of Science of the United States of America, 102: 7465-7469.

ROOT, T.L.; PRICE, J.T.; HALL, K.R.; SCHNEIDER, S.H.;
ROSENZWEIGK, C. \& POUNDS, J.A. 2003. Fingerprints of global warming on wild animals and plants. Nature, 421: 57-60.

RUIZ, D.; MORENO, H.A.; GUTIÉRREZ, M.E. \& ZAPATA, P.A. 2008. Changing climate and endangered high mountain ecosystems in Colombia. Science of the Total Environment, 398: $122-132$

SALA, O.E.; CHAPIN III, F.S.; ARMESTO, J.J.; BERLOW, E.; BLOOMFIELD, J.; DIRZO, R.; HUBER-SANWALD, E.; HUENNEKE, L.F.; JACKSON, R.B.; KINZIG, A.; LEEMANS, R.; LODGE, D.M.; MOONEY, H.A.; OESTERHELD, M.; POFF, N.L.; SYKES, M.T.; WALKER, B.H.; WALKER, M. \& WALL, D.H. 2000. Global Biodiversity Scenarios for the Year 2100. Science, 287: 1770-1774.

SALAZAR, L.F.; NOBRE, C.A. \& OYAMA, M.D. 2007. Climate change consequences on the biome distribution in tropical South America. Geophysical Research Letters 34: L09708.1-L09708.6. SANTILLI, M.; MOUTINHO, P.; SCHWARTZMAN, S.; NEPSTAD, D.; CURRAN, L. \& NOBRE, C. 2005. Tropical deforestation and the Kyoto Protocol. Climatic Change, 71: 267-276.

SCHRÖTER, D.; CRAMER, W.; LEEMASN, R.; PRENTICE, I.C.; ARAÚJO, M.B.; ARNELL, N.W.; BONDEAU, A.; BUGMANN, H.; CARTER, T.R.; GRACIA, C.A.; DE LA VEGALEINERT, A.C.; ERHARD, M.; EWERT, F.; GLENDINING, M.; HOUSE, J.I.; KANKAANPÄÄ, S.; KLEIN, R.J.T.; LAVOREL, S.; LINDNER, M.; METZGER, M.J.; MEYER, J.; MITCHELL, T.D.; REGINSTER, I.; ROUNSEVELL，M.; SABATÉ, S.; SITCH, S.; SMITH, B.; SMITH, J.F.; SMITH, P.; SYKES, M.T.; THONICKE, K.; THUILLER, W.; TUCK, G.; ZAEHLE, S. \& S; ZIERL, B. 2005. Ecosystem service supply and vulnerability to global change in Europe. Science, 310: 1333-1337.

SIQUEIRA, M.F. \& PETERSON, A.T. 2003. Consequences of global climate change for geographic distributions of Cerrado tree species. Biota Neotropica, 3(2): http://www.biotaneotropica. org.br/v3n2/pt/abstract?article+BN00803022003 (acesso em 18/11/2008).

SKOV, F. \& SVENNING, J.C. 2004. Potential impact of climatic change on the distribution of forest herbs in Europe. Ecography, 27: 366-380.

SOARES-FILHO, B.S.; DIETZSCH, L.; MOUTINHO, P.; FALIERI, A.; RODRIGUES, H.; PINTO, E.; MARETTI, C.C.; SUASSUNA, K.; SCARAMUZZA, C.A.M. \& ARAÚJO, F.V. 2008. Redução de emissões de carbono associadas ao desmatamento no Brasil: o papel do Programa Áreas Protegidas da Amazônia (Arpa). IPAM, Brasília. 32p. 
STERN, N. 2007. The Economics of Climate Change: the Stern Review. Cambridge University Press, Cambridge. 712p.

SVENNING, J.C. \& CONDIT, R. 2008. Biodiversity in a Warmer World. Science, 322: 206-207.

THOMAS, C.D.; CAMERON, A.; GREEN, R.E.; BAKLENES, M.; BEAUMONT, L.J.; COLLINGHAM, Y.C.; ERASMUS, B.F.N.; SIQUEIRA, M.F.; GRAIGNER, A.; HANNAH, L.; HUGHES, L.; HUNTLEY, B.; van JAARSVELD, A.S.; MIDGLEY, G.F.; MILES, L.; ORTEGA-HUERTA, M.A.; PETERSON, A.T.; PHILLIPS, O.L. \& WILLIAMS, S.E. 2004. Extinction risk from climate change. Nature, 427: 145-148.

THOMAS, C.D. \& LENNON, J.J. 1999. Birds extend their ranges northwards. Nature, 399: 213-213.

THOMAS, C.D.; BODSWORTH, E.J.; WILSON, R.J.; SIMMONS, A.D.; DAVIES, Z.G.; MUSCHE, M. \& CONRADT, L. 2001. Ecological and evolutionary processes at expanding range margins. Nature, 411: 577-581.

THUILLER, W. 2004. Patterns and uncertainties of species' range shifts under climate change. Global Change Biology, 10: 2020-2027.

THUILlER, W.; ALBERT, C.; ARAÚJO, M.B.; BERRY, P.M.; CABEZA, M.; GUISAN, A.; HICKLER, T.; MIDGLEY, G.F.; PATERSON, J.; SCHURR, F.M.; SYKES, M.T. \& ZIMMERMANN, N.E. 2008. Predicting global change impacts on plant species' distributions: Future challenges. Perspectives in Plant Ecology, Evolution and Systematics, 9: 137-152.

THUILLER, W.; BROENNIMANN, O.; HUGHES, G.O.; ALKEMADE, J.R.M.; MIDGLEY, G.F. \& CORSI, F. 2006. Vulnerability of African mammals to anthropogenic climate change under conservative land transformation assumptions. Global Change Biology, 12: 424-440.

THUILLER, W.; LAVOREL, S.; ARAÚJO, M.B.; SYKES, M.T. \& PRENTICE, I.C. 2005. Climate change threats to plant diversity in Europe. Proceeding of the National Academy of Science of the United States of America, 102: 8245-8250.

TØTTRUP, A.P.; THORUP, K. \& RAHBEK, C. 2006. Patterns of change in timing of spring migration in North European songbird populations. Journal of Avian Biology, 37: 84-92.

UN (UNITED NATIONS). 2005. Framework Convention on Climate Change (UNFCCC), Reducing emissions from deforestation in developing countries: Approaches to stimulate action. Conference of the Parties, Montreal, 28 November to 9 December 2005. FCCC/CP/2005/L.2, UNFCCC, Bonn, 2005. http://unfecc.int/ resource/docs/2005/cop11/eng/102.pdf (acesso em 28/10/2008).
VIANA, V.\& CAMPOS, M.T. 2007. Bolsa Floresta: Recompensa para Quem Conserva a Floresta em Pé. Secretaria do Estado do Meio Ambiente e Desenvolvimento Sustentável (SDS), Manaus, Brasil. 13p.

VISSER, M.E. \& HOLLEMAN, L.J.M. 2001. Warmer springs disrupt the synchrony of oak and winter moth phenology. Proceeding of the Royal Society of London B, 268: 289-294.

VISSER, M.E.; VAN NOORDWIJK, A.J.; TINBERGEN, J.M. \& LESSELLS, C.M. 1998. Warmer springs lead to mistimed reproduction in great tits (Parus major). Proceedings of the Royal Society of London B, 265: 1867-1870.

WALTHER, G.R.; BERGER, S. \& SYKES, M.T. 2005. An ecological "footprint" of climate change. Proceeding of the Royal Society of London B, 272: 1427-1432.

WALTHER, G.R.; POST, E.; CONVEY, P.; MENZEL, A.; PARMESAN, C.; BEEBEE, T.J.C.; FROMETIN, J.M.; HOEGHGULDBERG, O. \& BAIRLEIN, F. 2002. Ecological responses to recent climate change. Nature, 416: 389-395.

WHITE, M.A.; BRUNSELL, N. \& SCHWARTZ, M.D. 2003. Vegetation phenology in global change studies. Pp. 453-466. In: M.D. Schwartz (ed.). Phenology: An Integrative Environmental Science. Kluwer Academic Publishers, Dordrecht. 606p.

WILKINSON, C. \& SOUTER, D. 2008. Status of Caribbean coral reefs after bleaching and hurricanes in 2005. Global Coral Reef Monitoring Network, and Reef and Rainforest Research Centre, Townsville, USA. http://coralreefwatch.noaa.gov/caribbean2005 (acesso em 28/10/2008).

WILLIAMS, J.W.; SHUMAN, B.N.; WEBB III, T.; BARTLEIN, P.J. \& LEDUC, P.L. 2004. Late-Quaternary vegetation dynamics in North America: scaling from taxa to biomes. Ecological Monographs, 74(2): 309-334.

WILSON, R.J.; GUTIERREZ, D.; GUTIERREZ, J.; MARTÍNEZ, D.; AGUDO, R. \& MONSERRAT, V.J. 2005. Changes to the elevational limits and extent of species ranges associated with climate change. Ecolological Letters, 8: 1138-1146.

YOUNG, C.E.F.; KHAIR, A.; SIMOENS, L.A. \& MACKNIGHT, V. 2007. Pacto pela valorização da floresta e pela redução do desmatamento na Amazônia brasileira: Fundamentos econômicos da proposta de pacto nacional pela valorização da floresta e pelo fim do desmatamento na floresta Amazônica. Relatório Final. Greenpeace Brasil. http://www.greenpeace.org/raw/content/brasil/documentos/ amazonia/fundamentos-econ-micos-da-prop-2.pdf (acesso em 18/11/2008). 
ZAVALETA, E.S.; SHAW, M.R.; CHIARIELLO, N.R.; THOMAS, B.D.; CLELAND, E.E.; FIELD, C.B. \& MOONEY, H.A. 2003. Grassland responses to three years of elevated temperature, $\mathrm{CO}_{2}$, precipitation, and $\mathrm{N}$ deposition. Ecological Monographs, 73: 585-604.

Submetido em 13/12/2008. Aceito em 05/01/2009. 